\title{
Minimisation of total delay in two-way coordinated arterials
}

\author{
L. D’Acierno ${ }^{1}$, G. De Luca $^{2}$ \& M. Gallo ${ }^{2}$ \\ ${ }^{1}$ Department of Civil, Architectural and Environmental Engineering, \\ 'Federico II' University of Naples, Italy \\ ${ }^{2}$ Department of Engineering, University of Sannio (Benevento), Italy
}

\begin{abstract}
Coordination of the signalised intersections of an arterial is a widely studied problem, with the aim being to find optimal offsets, when the other control parameters (cycle time, effective greens, etc.) are designed. Optimal offsets are generally those that are able to minimise the stops at intersections for vehicles travelling along the coordinated arterial or to maximise bandwidth. While the problem is very simple for one-way arterials, it is more complex for two-way arterials where the problem is usually approached as one of bandwidth maximisation. However, the latter does not ensure minimum total delay (or total travel time) on the network. In this paper we study the problem of coordinating two-way signalised arterials with a view to minimising total delay, using a microsimulation approach to explore the solution set. Initial results show that the objective function of the problem is not convex and that several local optima can be identified.
\end{abstract}

Keywords: arterial coordination, traffic-lights, road traffic, microsimulation.

\section{Introduction}

Coordinating the signal settings of an arterial is a control strategy to minimise travel delays on a main road with multiple consecutive intersections. Since in urban networks junction delays constitute a substantial proportion of running times, the importance of proper coordination appears evident. Over the years several techniques and models have been developed for optimising signal settings, and three main problems have emerged: (a) single junction optimisation; (b) arterial optimisation/coordination; (c) multiple junction 
optimisation (signal network control). In this paper we tackle problem (b), focusing on how to minimise the total delay of a two-way arterial. For the literature on the multiple junction optimisation problem (see Cascetta et al. [1] and D'Acierno et al. [2]).

Of the many papers, books, handbooks and software programs that have been proposed, some tackle the problem using simulation-based models, while others propose to use analytical models. Examples of simulation-based models are TRANSYT-7F [3, 4] and SIGOP III [5] while analytical models have been proposed by Gartner et al. [6] and Liu and Chang [7].

The solution of the single arterial coordination problem is simple if the arterial is one-way: in this case optimal green offsets can be calculated according to distance between intersections and average flow speed, always obtaining the ideal coordination corresponding to the maximum bandwidth (defined as the time interval during which the vehicles are able to travel on the road without any stops at intersections). The details can be found, for instance, in [8, 9] and the solution methods are widely used in practical applications. In fig. 1 a space/time diagram shows the ideal coordination and the parameters of the problem are identified; in this case the (maximum) bandwidth is equal to the effective green time and its capacity is equal to the product of the same bandwidth and the saturation flow.

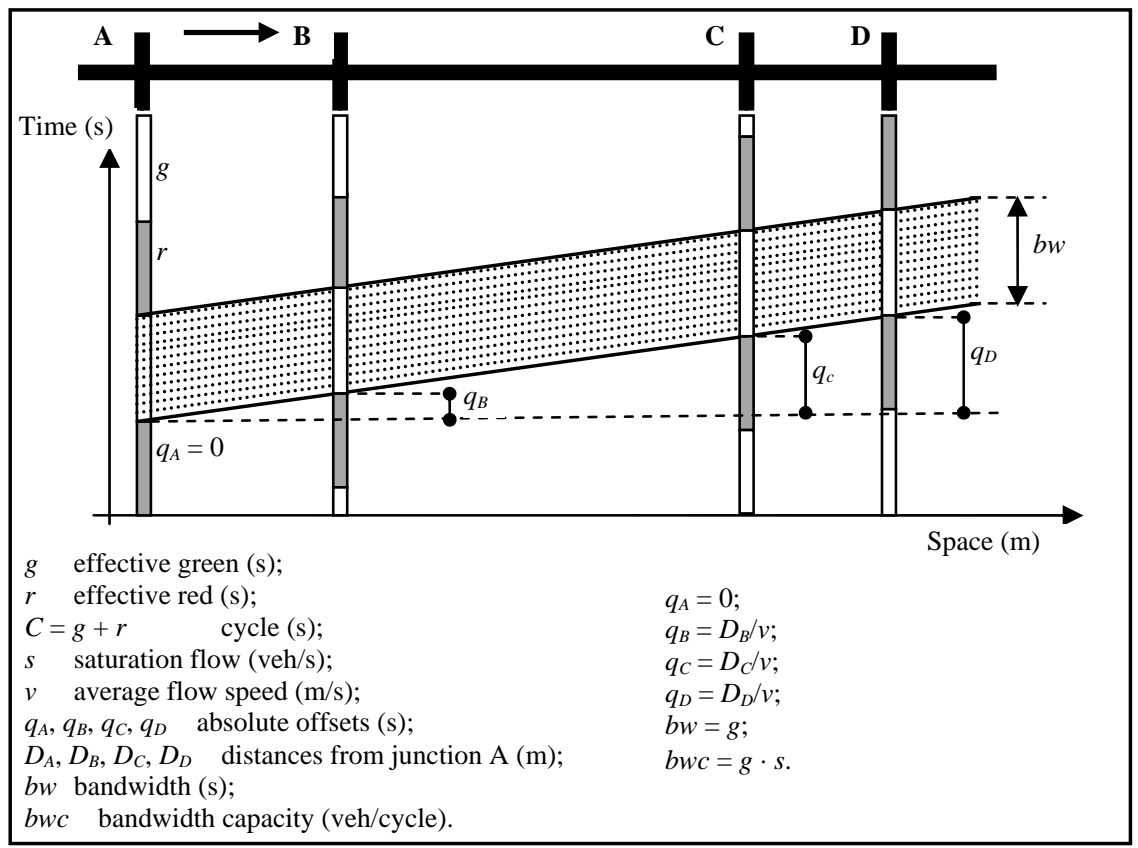

Figure 1: Ideal coordination of a one-way arterial and parameters. 
For two-way arterials the coordination problem is more complex since it is not always possible to obtain the ideal coordination in both directions. It may be stated that the ideal coordination in both directions is possible only if:

$$
C \leq 2 D / v_{\min }
$$

where:

$C \quad$ is the cycle time (s);

$D$ is the distance between two intersections (m);

$v_{\min }$ is the minimum average speed of traffic flow $(\mathrm{m} / \mathrm{s})$.

It may be verified that this condition is compatible with feasible cycle lengths only when the distances between intersections are significant, that is where the effects of coordination produce lower benefits.

In real applications only one direction is usually coordinated (the one with the higher traffic flow); this case is solved with the same methods adopted for oneway arterials.

In the literature, the bandwidth maximisation problem was introduced partly to maximise the sum of bandwidths in both directions (see Morgan and Little [10]; Little [11]; Inose and Hamada [12]). Papola and Accattatis [13], Papola [14] and Papola and Fusco [15] studied the problem assuming the cycle length and progression speed as fixed; such variables are considered design variables by Bass [16] and Little et al. [17] who proposed the MAXBAND model. An evolution of the MAXBAND model was the MULTIBAND model (Gartner et al. [18], Gartner and Hou [19]), which was more generalised by Stamadiatis and Gartner [20].

The solution of the bandwidth maximisation problem may not correspond to the minimum total delay on the network. In this paper, we study the problem of minimising the total delay of an arterial by adopting a microsimulation model to evaluate arterial performance and explore the solution set.

The paper is organised as follows: Section 2 formulates the optimisation model and Section 3 describes the solution algorithm; Section 4 summarises the results obtained in a real case study; finally, conclusions are drawn and research prospects outlined in Section 5.

\section{Model formulation}

We consider a two-way arterial where all intersections are signalised; we assume that the cycle time, $C$, and the effective green times, $g_{c}$ and $g_{n c}$, are calculated as a function of known traffic flows (subscripts $c$ and $n c$ refer respectively to the coordinated and non-coordinated phase), for instance with the well-known Webster method [21]. We recall that in this case all coordinated junctions should have the same cycle time and usually the same effective greens. Hence the Webster method is used by considering the maximum values for the flow on the saturation flow ratios of each phase. 
We optimise the total delay on the arterial, $t d$, that is the sum of the delays at all approaches to the arterial's junctions. Obviously, the total delay depends on the offsets of the junction, $\boldsymbol{q}$. The total delay is calculated by a microsimulation model.

The optimisation model can be formulated as follows:

$$
\boldsymbol{q}^{*}=\operatorname{Arg}_{\boldsymbol{q}} \min t d(\boldsymbol{q})
$$

s.t.:

$$
\begin{gathered}
\mathbf{0} \leq \boldsymbol{q}<\boldsymbol{C} \\
t d(\boldsymbol{q})=M S(\boldsymbol{q})
\end{gathered}
$$

where:

$\boldsymbol{q}$ is the vector of the offsets;

$\boldsymbol{q}^{*} \quad$ is the optimal value for $\boldsymbol{q}$;

$\mathbf{0}$ is the zero vector (a vector with the same dimension of the $\boldsymbol{q}$ vector with all components equal to 0 );

$\boldsymbol{C} \quad$ is the cycle vector (a vector with the same dimension of the $\boldsymbol{q}$ vector with all components equal to $C$ );

$M S(\boldsymbol{q})$ indicates the microsimulation model that is able to estimate the total delay as a function of the offsets.

This is a constrained non-linear optimisation model requiring a microsimulation model to be set up to estimate the value of the objective function.

\section{Solution algorithm}

In order to solve the optimisation model formulated in the previous section, we propose a multi-start neighbourhood search algorithm. Neighbourhood search methods are widely used for solving operation research problems, sometimes as subroutines of more complex solution algorithms. Some applications to network design problems can be found in Gallo et al. [22-25].

The neighbourhood search (NS) algorithm searches for the local optimum nearest to the starting solution. Having set $\boldsymbol{q}^{i t}$ as the solution at iteration it, its neighbourhood is the set of solutions, $N\left(\boldsymbol{q}^{i t}\right)$, that can be generated from the current solution by a single move, obtained by changing the value of an offset with a predetermined step (for instance $+5 \mathrm{~s}$ or $-5 \mathrm{~s}$ ). Since we have to set to 0 the value of an offset (generally the offset of the first or last junction of the arterial) the number of variables are $n_{i n t}-1$, where $n_{i n t}$ is the number of coordinated intersections and a neighbourhood contains $2\left(n_{\text {int }}-1\right)$ solutions.

Formally, neighbourhood generation can be expressed as:

where:

$$
N\left(\boldsymbol{q}^{i t}\right) \supseteq \boldsymbol{q}_{z}^{i t+1}: \boldsymbol{q}_{z}^{i t+1} \in\left\{\boldsymbol{q}^{i t+1}{ }_{N+}, \boldsymbol{q}^{i t+1}{ }_{N-}\right\}
$$

$\boldsymbol{q}^{i+1}{ }^{++}$:

for each int 


$$
\begin{aligned}
& \text { if } q_{i, \text {,int }}^{i t}=1 \\
& \quad q_{i^{\prime}+1, i n t}^{i t+1}=1 \\
& q_{i, \text { int }}^{i t+1}=0 \quad \forall i \neq i^{\prime}+1
\end{aligned}
$$

repeat

$\boldsymbol{q}^{i t+1}{ }^{-1}$ :

$$
\begin{aligned}
& \text { for each int } \\
& \text { if } q^{i t}{ }^{i, i n t}=1 \\
& q^{i t+1}{ }^{\prime}-1, i n t=1 \\
& q^{i t+1}{ }_{i, i n t}=0 \quad \forall i \neq i^{\prime}-1
\end{aligned}
$$

An NS algorithm can adopt two different approaches for generating the next solution:

- the steepest descent method, which examines and evaluates all neighbouring solutions, $N\left(\boldsymbol{q}^{i t}\right)$, and chooses the best solution as the next one;

- $\quad$ the random descent method, which randomly extracts a solution from the neighbourhood, $N\left(\boldsymbol{q}^{i t}\right)$, and evaluates only its objective function; if the new solution is better than the current one, it becomes the current solution; otherwise, another neighbourhood solution is randomly extracted and so on. If no neighbourhood solutions improve the objective function, the search stops and the last solution is a local optimum.

In the steepest descent method the next solution will be generated as the best feasible solution included in $N\left(\boldsymbol{q}^{i t}\right)$ :

$$
\boldsymbol{q}_{S D}^{i t+1}=\arg \boldsymbol{q}_{z}^{i t+1} \in N\left(\boldsymbol{q}^{i t}\right) \min t d\left(\boldsymbol{q}_{z}^{i t+1}\right)
$$

and the algorithm will stop if:

$$
t d\left(\boldsymbol{q}^{i t+1}\right) \geq t d\left(\boldsymbol{q}^{i t}\right)
$$

Instead, in the random descent method the next solution is the first randomly extracted solution that reduces the objective function value. In our problem, since the variables are not numerous, we prefer to use the steepest descent method.

The necessity of a multi-start procedure is due to the non-convexity of the objective function, as highlighted by the numerical results (see next section); in particular, we propose to start the NS from three different solutions:

1. the zero solution, where all offsets are set to 0 ;

2. solution A, where the offsets are designed so as to ideally coordinate a direction of the arterial;

3. solution $\mathrm{B}$, where the offsets are designed so as to ideally coordinate the other direction of the arterial.

However, in most cases should there be a large number of offsets to define, then other starting solutions can be adopted. 


\section{Numerical results}

The proposed model and algorithm were tested on a real case, namely an arterial in the urban network of Benevento (Italy). Although the arterial has four signalised intersections, two are so close that they have to have the same offset. Considering that the offset of an intersection has to be set to 0 , the problem has only 2 variables, $q_{1}$ and $q_{2}$.

Traffic flows in the peak-hour on the links of the arterial were obtained by specific surveys; Figure 2 reports the arterial and the hourly traffic flows.

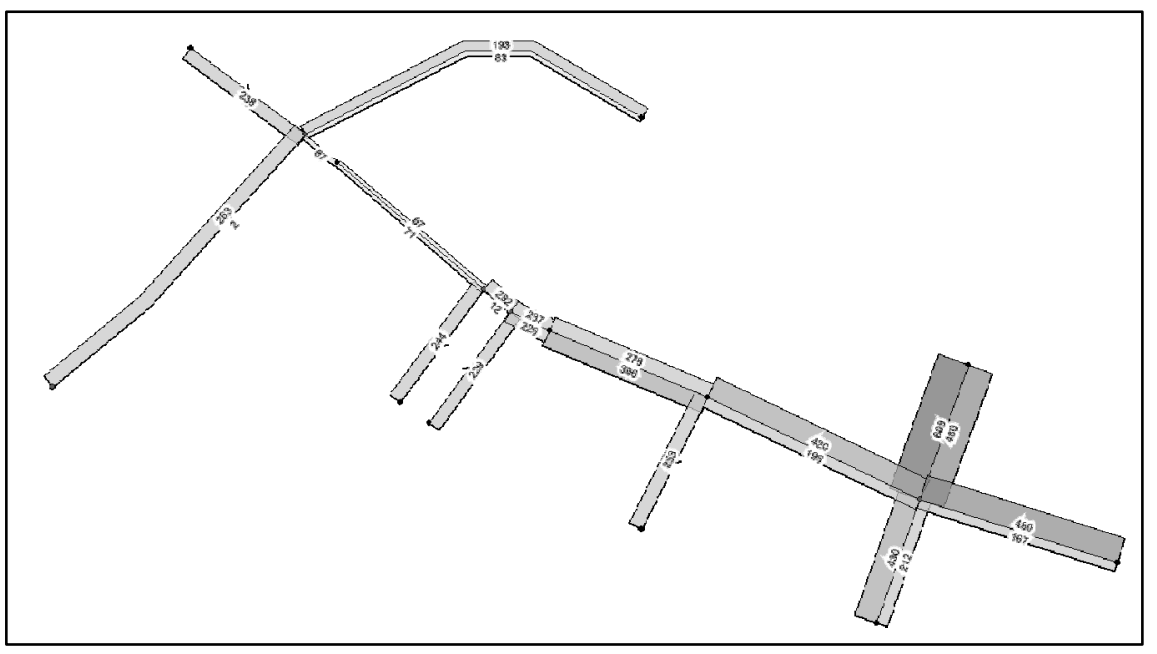

Figure 2: $\quad$ Test case.

The cycle time, $C$, calculated by the Webster method, is equal to $60 \mathrm{~s}$ and the effective green times are $g_{c}=23 \mathrm{~s}$ and $g_{n c}=29 \mathrm{~s}$. The microsimulation model used for estimating delays is the CORSIM. The parameters of the microsimulation model were calibrated by surveys on the Benevento network.

The multi-start neighbourhood search method leads to three different solutions, one for each starting point; in Table 1 the steps of the algorithms are summarised. Note that the two offsets to design (a third offset is set to 0) are identified by $q_{1}$ and $q_{2}$ and that the total delay is expressed in minutes.

Table 1: $\quad$ Multi-start neighbourhood search algorithm steps.

\begin{tabular}{|c|c|c|c|c|c|c|c|c|c|}
\hline \multirow{2}{*}{ Iteration } & \multicolumn{3}{|c|}{ Start. Solution 0} & \multicolumn{3}{|c|}{ Start. Solution A } & \multicolumn{3}{|c|}{ Start. Solution B } \\
\hline & $q_{1}$ & $q_{2}$ & $t d(\boldsymbol{q})$ & $q_{1}$ & $q_{2}$ & $t d(\boldsymbol{q})$ & $q_{1}$ & $q_{2}$ & $t d(\mathbf{q})$ \\
\hline 1 & 0 & 0 & 14.54 & 40 & 25 & 15.83 & 35 & 20 & 15.31 \\
\hline 2 & 55 & 0 & 13.88 & 45 & 25 & 14.55 & 40 & 20 & 14.57 \\
\hline 3 & 55 & 5 & 13.80 & 50 & 25 & 13.86 & 40 & 15 & 14.25 \\
\hline 4 & 50 & 5 & 13.61 & 50 & 30 & 13.71 & 45 & 15 & 13.96 \\
\hline 5 & 50 & 10 & 13.54 & 55 & 30 & 13.06 & 50 & 15 & 13.62 \\
\hline 6 & & & & & & & 55 & 15 & 13.46 \\
\hline
\end{tabular}


An exhaustive search shows that the local optimum obtained starting from the solution $\mathrm{A}\left(q_{1}=55 \mathrm{~s}\right.$ and $\left.q_{2}=30 \mathrm{~s}\right)$ is also the global optimum. Interestingly, solution $\mathrm{A}$ is the one that gives the ideal coordination for the direction where the traffic flows are higher.

Table 2 shows the results of the exhaustive search and Figure 2 the shape of the objective function. Figure 3 shows the steps of the multi-start neighbourhood search.

Table 2: $\quad$ Results of the exhaustive search.

\begin{tabular}{|c|c|c|c|c|c|c|c|c|c|c|c|c|}
\hline $\boldsymbol{q}_{\mathbf{1}} \mid \boldsymbol{q}_{\mathbf{2}}$ & $\mathbf{0}$ & $\mathbf{5}$ & $\mathbf{1 0}$ & $\mathbf{1 5}$ & $\mathbf{2 0}$ & $\mathbf{2 5}$ & $\mathbf{3 0}$ & $\mathbf{3 5}$ & $\mathbf{4 0}$ & $\mathbf{4 5}$ & $\mathbf{5 0}$ & $\mathbf{5 5}$ \\
\hline $\mathbf{0}$ & 14.54 & 14.07 & 13.90 & 13.47 & 13.56 & 14.14 & 13.48 & 13.40 & 14.11 & 14.88 & 13.97 & 14.55 \\
\hline $\mathbf{5}$ & 15.14 & 14.56 & 14.24 & 13.67 & 13.79 & 14.09 & 13.64 & 13.67 & 14.14 & 14.12 & 13.90 & 14.35 \\
\hline $\mathbf{1 0}$ & 15.33 & 14.75 & 14.95 & 13.95 & 14.09 & 14.38 & 13.94 & 13.90 & 14.24 & 14.69 & 13.93 & 14.41 \\
\hline $\mathbf{1 5}$ & 14.45 & 14.51 & 14.47 & 14.30 & 14.59 & 14.83 & 13.73 & 13.79 & 14.29 & 14.56 & 13.92 & 14.20 \\
\hline $\mathbf{2 0}$ & 14.52 & 14.78 & 14.94 & 14.62 & 14.86 & 15.22 & 14.31 & 14.16 & 14.62 & 14.34 & 13.95 & 14.58 \\
\hline $\mathbf{2 5}$ & 14.95 & 14.89 & 15.00 & 15.08 & 15.61 & 15.68 & 14.75 & 15.02 & 14.96 & 14.80 & 14.53 & 14.54 \\
\hline $\mathbf{3 0}$ & 14.54 & 14.68 & 14.60 & 14.73 & 15.65 & 16.11 & 15.10 & 14.79 & 14.84 & 14.37 & 14.05 & 14.58 \\
\hline $\mathbf{3 5}$ & 14.57 & 14.96 & 14.84 & 14.92 & 15.31 & 16.31 & 15.16 & 14.96 & 15.26 & 14.96 & 14.61 & 14.84 \\
\hline $\mathbf{4 0}$ & 14.27 & 14.64 & 14.39 & 14.25 & 14.57 & 15.83 & 14.92 & 15.14 & 15.27 & 14.87 & 13.97 & 14.51 \\
\hline $\mathbf{4 5}$ & 14.04 & 14.19 & 14.11 & 13.96 & 14.38 & 14.55 & 14.77 & 14.92 & 15.35 & 15.10 & 14.36 & 14.41 \\
\hline $\mathbf{5 0}$ & 13.93 & 13.61 & $\mathbf{1 3 . 5 4}$ & 13.62 & 13.83 & 13.86 & 13.71 & 13.54 & 14.64 & 15.03 & 14.18 & 14.32 \\
\hline $\mathbf{5 5}$ & 13.88 & 13.80 & 13.57 & $\mathbf{1 3 . 4 6}$ & 13.58 & 14.01 & $\mathbf{1 3 . 0 6}$ & 13.50 & 14.18 & 14.67 & 14.55 & 14.52 \\
\hline
\end{tabular}

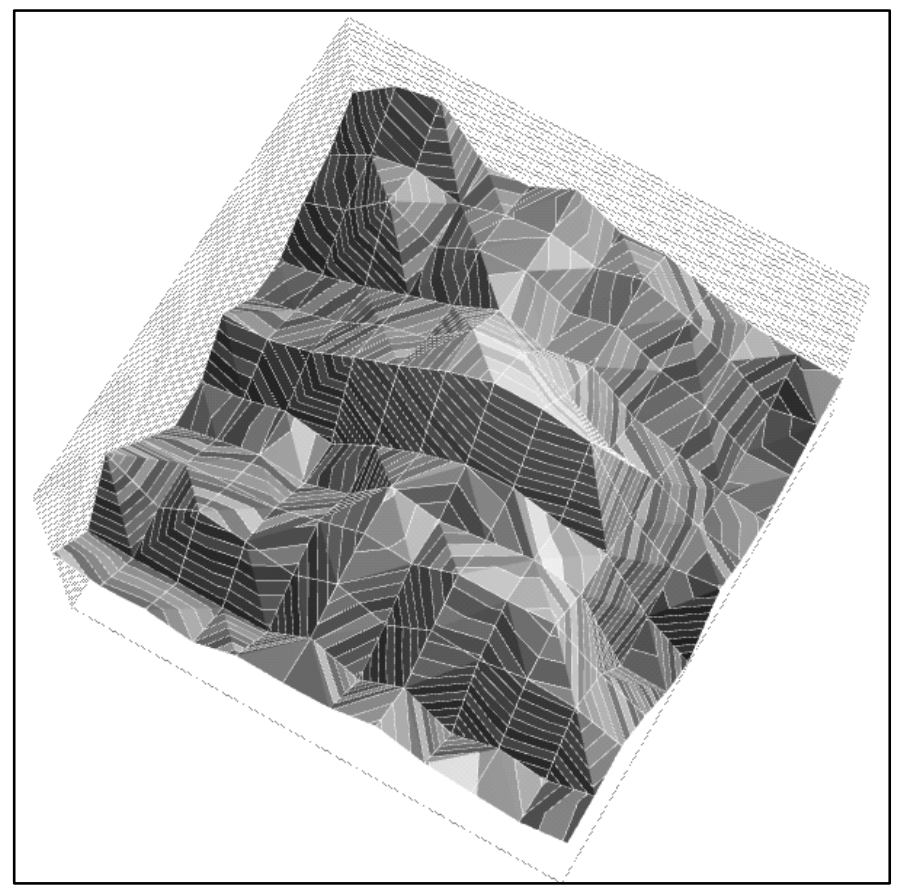

Figure 3: The shape of the objective function. 
On examining the exhaustive search it can be noted that the best solution obtained by the multi-start neighbourhood search method is also the global optimum. Moreover, the three local optimal solutions have similar values of $q_{1}$ (in two cases the value is the same).

Examination of Figure 2 shows that the objective function is not convex: even if some oscillations of the objective function values may be due to the stochastic features of the microsimulation model, several zones of the space of solutions where the objective function values are lower can be identified, corresponding to several local optima.

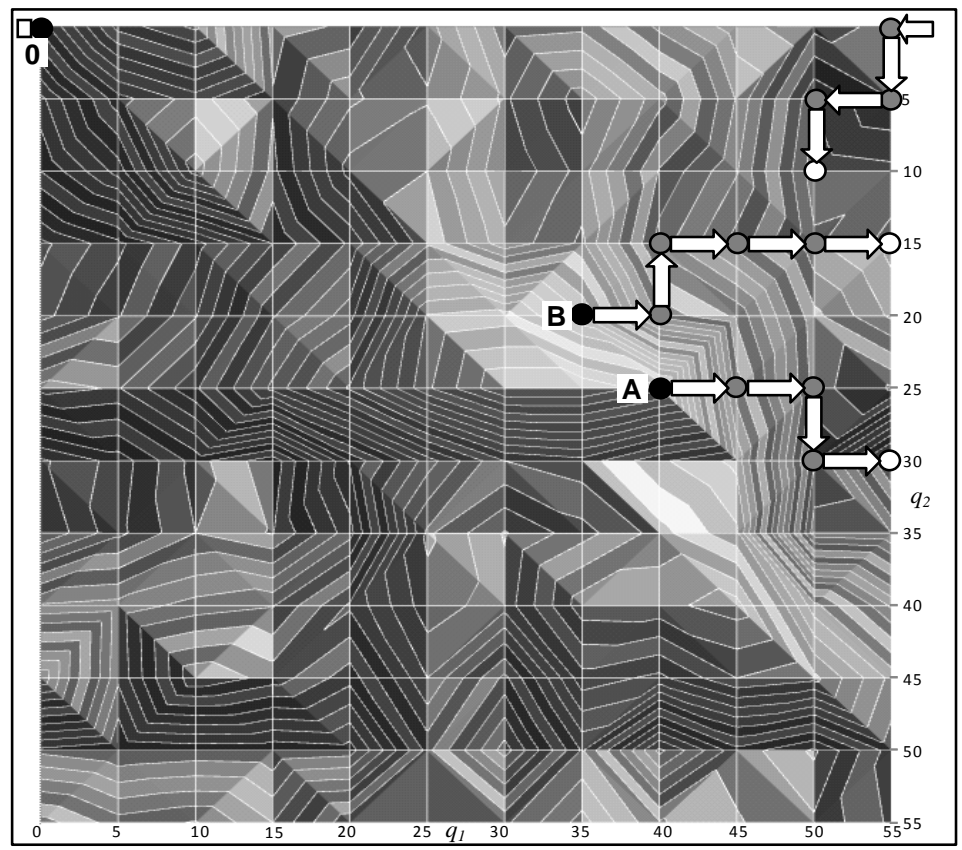

Figure 4: $\quad$ Steps of the multi-start neighbourhood search.

Table 3: $\quad$ Results of the densified search.

\begin{tabular}{|c|c|c|c|c|c|c|c|c|c|}
\hline $\boldsymbol{q}_{\mathbf{1}} \backslash \boldsymbol{q}_{\mathbf{2}}$ & $\mathbf{2 6}$ & $\mathbf{2 7}$ & $\mathbf{2 8}$ & $\mathbf{2 9}$ & $\mathbf{3 0}$ & $\mathbf{3 1}$ & $\mathbf{3 2}$ & $\mathbf{3 3}$ & $\mathbf{3 4}$ \\
\hline $\mathbf{4 6}$ & 13.97 & 14.41 & 14.16 & 13.99 & 14.24 & 14.61 & 14.65 & 14.53 & 14.33 \\
\hline $\mathbf{4 7}$ & 14.14 & 14.52 & 14.39 & 14.11 & 14.27 & 14.30 & 14.31 & 14.24 & 14.31 \\
\hline $\mathbf{4 8}$ & 14.02 & 14.23 & 14.51 & 14.19 & 14.27 & 14.05 & 14.04 & 14.19 & 14.36 \\
\hline $\mathbf{4 9}$ & 14.05 & 14.07 & 13.89 & 13.92 & 13.81 & 13.56 & 13.69 & 13.64 & 13.97 \\
\hline $\mathbf{5 0}$ & 13.97 & 13.75 & 13.82 & 14.00 & $\mathbf{1 3 . 0 6}$ & 13.56 & 13.72 & 13.89 & 13.46 \\
\hline $\mathbf{5 1}$ & 14.20 & 14.34 & 13.83 & 13.85 & 13.72 & 13.84 & 13.68 & 13.78 & 13.73 \\
\hline $\mathbf{5 2}$ & 14.07 & 14.13 & 13.71 & 13.69 & 13.52 & 13.56 & 13.51 & 13.37 & 13.59 \\
\hline $\mathbf{5 3}$ & 14.03 & 14.06 & 13.68 & 14.06 & 13.86 & 13.91 & 13.36 & 13.49 & 13.58 \\
\hline $\mathbf{5 4}$ & 14.39 & 14.07 & 13.64 & 13.48 & 13.48 & 13.48 & 13.60 & 13.48 & 13.79 \\
\hline
\end{tabular}


For the best solution obtained we densify the search, considering a grid with one second steps (see Table 3). In this particular case the best solution is the one already found.

An exhaustive search is possible in this particular test case since we have only two offsets to design. In longer arterials where there may be up to 8-10 offsets to design, an exhaustive search is not possible with acceptable computing times. However, the proposed multi-start neighbourhood search is able to produce some local optima with acceptable computing times.

\section{Conclusions and research prospects}

In this paper we studied the problem of optimising the offsets of a two-way arterial so as to minimise total delays. We proposed a model and an algorithm for solving the problem. The test performed on a real case showed that the objective function is not convex; the proposed multi-start neighbourhood search algorithm allows some several local optima to be generated. In the specific test case, where also an exhaustive search was developed, one of the local optima is also the global optimum.

A possible line for future research would be to consider several arterials in the whole network, given that traffic flows also change with the change in coordination parameters. In this case, cycle times and effective green times should also be updated at each iteration.

\section{Acknowledgement}

This paper is partially supported by the Italian MIUR under PRIN2009 grant no. 2009EP3S42_002.

\section{References}

[1] Cascetta, E., Gallo, M. and Montella B., Models and algorithms for the optimization of signal settings on urban networks with stochastic assignment. Annals of Operations Research, 144(1), pp. 301-328, 2006.

[2] D’Acierno, L., Gallo, M. and Montella B., An Ant Colony Optimisation algorithm for solving the asymmetric traffic assignment problem. European Journal of Operational Research, 217(2), pp. 459-469, 2012.

[3] Robertson, D.I., TRANSYT: traffic network study tool. 4th International Symposium on the theory of traffic flow, Karlsruhe, Germany, 1968.

[4] Wallace, C.E., Courage, K.G., Reaves, D.P., Shoene, G.W., Euler, G.W. and Wilbur, A., TRANSYT 7F. Technical Report for FHWA by Transportation Research Center, University of Florida, USA, 1988.

[5] Liebermann, E.B., Lai, J. and Elllington, R.E., SIGOP III Technical Report. FHWA, Washington D.C., USA, 1983. 
[6] Gartner, N.H., Little, J.D.C. and Gabbay, H., Optimization of traffic signal settings by mixed-integer linear programming. Part II: The network syncronization problem. Transportation Science, 9(4), pp. 344-363, 1975.

[7] Liu, Y. and Chang, G.L., An arterial signal optimization model for intersections experiencing queue spillback and lane blockage. Transportation Research Part C, 19(1), pp. 130-144, 2011.

[8] ITE, Traffic Engineering Handbook. 6th edition. Institute of Transportation Engineers, Washington D.C., USA, 2009.

[9] Roess, R.P., Prassas, E.S. and McShane, W.R., Traffic Engineering. 4th edition. Prentice-Hall, Upper Saddle River (NJ), USA, 2010.

[10] Morgan, J.T. and Little, J.D.C., Synchronizing traffic signals for maximal bandwidth, Operations Research, 12(6), pp. 896-912, 1964.

[11] Little, J.D.C, The synchronization of traffic signals by mixed integer linear programming. Operations Research, 14(4), pp. 568-594, 1966.

[12] Inose, H., and Hamada, T., Road Traffic Control. University of Tokyo Press, Tokyo, Japan, 1975.

[13] Papola, N., and Accattatis, F. Teoria del deflusso veicolare e suo impiego nella regolazione e progettazione- Parte II. Collana Atti di trasporti, 1989.

[14] Papola, N., Bandwidth maximization: split and unsplit solutions. Transportation Research Part B, 26(5), pp. 341-356, 1992.

[15] Papola, N., and Fusco, G., Maximal bandwidth problems: a new algorithm based on the properties of periodicity of the system. Transportation Research Part B, 32(4), pp. 277-288, 1998.

[16] Bass, K.A., Another look at bandwidth maximization. Transportation Research Record, 905, pp. 38-47, 1983.

[17] Little, J.D.C, Kelson, M.D. and Gartner, N.H., MAXBAND: a program for setting signals on arteries and triangular networks. Transportation Research Record, 795, pp. 40-46, 1981.

[18] Gartner, N.H., Assmann, S.F. and Lasaga, F.L., A multiband approach to arterial traffic signal optimization. Transportation Research Part B, 25(1), pp. 55-74, 1991.

[19] Gartner, N.H. and Hou, D.L., Performance evaluation of multiband progression method. Proceedings of seventh IFAC/IFORS Symposium on Transportation Systems: Theory and Application of Advanced Technology, 1994.

[20] Stamadiatis, C. and Gartner, N.H., MULTIBAND-96: a program for variable bandwidth progression optimization of multiarterial traffic networks. Transportation Research Record, 1554, pp. 9-17, 1996.

[21] Webster, V.F., Traffic signal settings. Road Research Technical Paper, 39, HMSO, London, United Kingdom, 1958.

[22] Gallo, M., D’Acierno, L. and Montella, B., A meta-heuristic approach for solving the Urban Network Design Problem. European Journal of Operational Research, 201(1), pp. 144-157, 2010.

[23] Gallo, M., Montella, B. and D’Acierno, L., The transit network design problem with elastic demand and internalisation of external costs: an 
application to rail frequency optimisation. Transportation Research Part C, 19(6), pp. 1276-1305, 2011.

[24] Gallo, M., D’Acierno, L. and Montella, B., A multimodal approach to bus frequency design. WIT Transactions on the Built Environment, 116, pp. 193-204, 2011.

[25] Gallo, M., D’Acierno, L. and Montella, B., A meta-heuristic algorithm for solving the road network design problem in regional contexts. Procedia Social and Behavioral Sciences, 54, pp. 84-95, 2012. 